Please do not remove this page

RMIT

UNIVERSITY

\title{
Spin guides and spin splitters: Waveguide analogies in one-dimensional spin chains
}

Makin, M; Cole, Jared; Hill, C; Greentree, Andrew

https://researchrepository.rmit.edu.au/esploro/outputs/9921858297901341/filesAndLinks?institution=61RMIT_INST\&index=null

Makin, M., Cole, J., Hill, C., \& Greentree, A. (2012). Spin guides and spin splitters: Waveguide analogies in one-dimensional spin chains. Physical Review Letters, 108(1), 017207-1-017207-5.

https://doi.org/10.1103/PhysRevLett.108.017207

Document Version: Published Version

Published Version: https://doi.org/10.1103/PhysRevLett.108.017207

Repository homepage: https://researchrepository.rmit.edu.au

(C) 2012 American Physical Society

Downloaded On 2023/04/27 01:25:37 +1000

Please do not remove this page 
Thank you for downloading this document from the RMIT Research Repository.

The RMIT Research Repository is an open access database showcasing the research outputs of RMIT University researchers.

RMIT Research Repository: http://researchbank.rmit.edu.au/

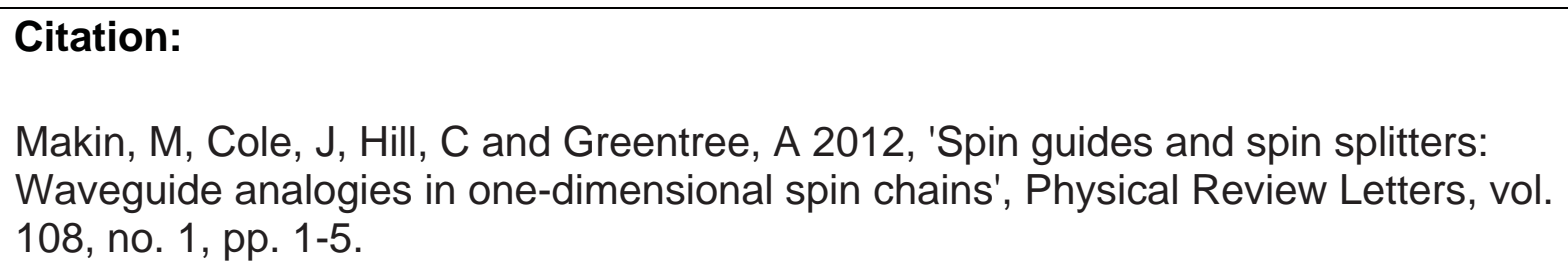

Version: Published Version

Copyright Statement:

(C) 2012 American Physical Society

Link to Published Version:

http://dx.doi.org/10.1103/PhysRevLett.108.017207 


\title{
Spin Guides and Spin Splitters: Waveguide Analogies in One-Dimensional Spin Chains
}

\author{
Melissa I. Makin, ${ }^{1}$ Jared H. Cole, ${ }^{2,3}$ Charles D. Hill, ${ }^{4}$ and Andrew D. Greentree ${ }^{1}$ \\ ${ }^{1}$ School of Physics, The University of Melbourne, Melbourne 3010, Australia \\ ${ }^{2}$ Chemical and Quantum Physics, School of Applied Sciences, RMIT University, Melbourne 3001, Australia \\ ${ }^{3}$ Institut für Theoretische Festkörperphysik and DFG-Center for Functional Nanostructures (CFN), \\ Karlsruher Institut für Technologie, 76128 Karlsruhe, Germany \\ ${ }^{4}$ ARC Centre for Quantum Computation and Communication Technology, School of Physics, The University of Melbourne, \\ Melbourne 3010, Australia
}

(Received 16 September 2011; published 5 January 2012)

\begin{abstract}
Here we show a mapping between waveguide theory and spin-chain transport, opening an alternative approach to solid-state quantum information transport. By applying temporally varying control profiles to a spin chain, we design a virtual waveguide or "spin guide" to conduct spin excitations along defined space-time trajectories of the chain. We show that the concepts of confinement, adiabatic bend loss, and beam splitting can be mapped from optical waveguide theory to spin guides, and hence to "spin splitters." Importantly, the spatial scale of applied control pulses is required to be large compared to the interspin spacing, thereby allowing the design of scalable control architectures.
\end{abstract}

PACS numbers: 75.10.Pq, 03.67.Hk, 05.60.Gg, 75.30.Ds

The application of quantum information science to technology promises to make a disruptive change to $21 \mathrm{st}$ century society, comparable to the computer and telecommunications revolutions of the 20th century. To this end, there is a pressing need to develop viable quantum networks. Here we focus on one quantum channel that is ideally suited to solid-state quantum computing: the onedimensional spin chain.

Spin-chain physics offers a rich phenomenology. There is a comprehensive review of the application of spin chains to quantum information processing by Bose [1]. In general, a spin chain is a one-dimensional array of spins that are closely spaced to facilitate strong spin-spin interactions, perhaps via dipole-dipole or exchange coupling. As the interspin spacing is typically on atomic or near atomic scales, individual addressability of the spins is either impossible or unscalable [2-5]. As a consequence of the restriction on local control, many innovative schemes have been studied for spin transport including schemes with uniform spins and control over just the ends of the chains (see Refs. [1,3-7]), parallel quantum channels [8,9], or with engineered coupling schemes [10,11]. There has also been related work in transport in coupled cavity systems [12-14].

Here we outline a distinct alternative to the problem of long-range quantum information transport inspired by optical waveguides [15]. We demonstrate that it is possible to create a virtual waveguide or "spin guide" in a onedimensional spin chain to guide individual spin excitations, magnons [16], as depicted in Fig. 1(a). The demonstration of this analogy is our central result.

Guiding of optical signals via waveguides and optical fiber is of vital importance to modern communications and sensing [17]. In addition, a vast array of devices have been developed in the waveguide space including splitters (fanout), Bragg mirrors, and fiber tapers. In optical waveguides, the propagation of light is confined in two dimensions by a refractive index profile, and in the simplest form, allowed to freely propagate in the third dimension. A schematic of an optical waveguide is shown in Fig. 1(b). Our analogy between spin-guide physics and waveguide technology shows that essentially all of the development in optical waveguides can be ported to the spin setting

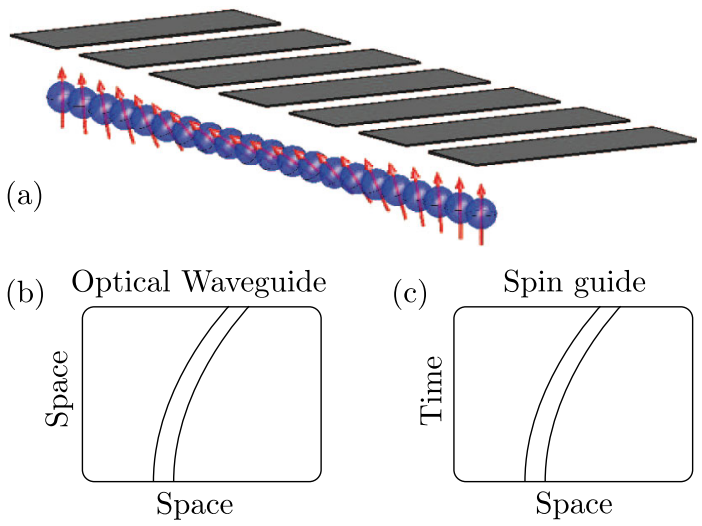

FIG. 1 (color online). (a) Schematic of a spin guide. A onedimensional line of spins is positioned below a gate array. The gate potentials are varied, breaking the translation symmetry of the chain, to define a spin guide capable of carrying an excitation. The size of the gates is expected to be much larger than the interspin spacing so that individual control of the spins is not possible. (b) A conventional waveguide is defined by a local change in the refractive index of a medium. This can be thought of as defining a two-dimensional pathway. (c) A spin guide is defined by a $1+1$ dimensional variation in the spin properties, mimicking the refractive index profile of an optical waveguide. 
opening a new vista for solid-state spin devices and a fundamentally new approach to the manipulation of excitations in spin chains.

To realize the analogy between a spin guide and an optical waveguide, we envisage a spatially and timevarying potential that is controllably swept across the one-dimensional spin chain. This potential breaks the degeneracy of the spins and is depicted schematically in Fig. 1(c). In essence, the two-dimensional waveguide is replaced by a $1+1$ dimensional spin guide, where the time axis for the spin guide should be thought of as equivalent to the spatial propagation axis for the waveguide. Note that although optical waveguides can usually house many excitations, we only consider the one-magnon subspace here.

There is considerable flexibility about the choice of applied magnetic field, and this necessarily affects the details of propagation, but the central results of the analogy are unchanged if alternate potentials are chosen. For simplicity we choose a magnetic field defined by a PöschlTeller (PT) potential, for which numerous analytical results are known [18-20], acting on a one-dimensional Heisenberg chain. In general any potential that can be used for optical waveguiding can be translated into the spinguide model, and any spin chain that supports magnons used as the medium. By adiabatically varying the PT potential as a function of time, the magnon is guided through a space-time map in a fashion analogous to conventional optical waveguiding. It should be noted that the use of magnons with Gaussian spatial distributions [21], and adiabatic following of a locally applied control field [22,23], have been considered, but we are not aware of any scheme that has applied the physics of optical waveguiding to coherent evolution of a solid-state excitation as we describe here.

The Hamiltonian for a system of $N$ spin $1 / 2$ particles with an applied field is

$$
H=-J \sum_{n=1}^{N} \mathbf{S}_{n} \cdot \mathbf{S}_{n+1}-B(n, t) S_{n}^{z}
$$

where $J$ is the exchange interaction strength, $\mathbf{S}_{n}$ and $S_{n}^{z}$ are operators for the total spin and the $z$ projection, respectively, for spin $n$, and $B(n, t)$ is the time-varying magnetic field applied to spin $n$. As the control fields are slowly varying across the spin-spin separation, we can replace the discrete spin-chain Hamiltonian Eq. (1) with its continuum counterpart $\mathcal{H}$ and solve the Schrödinger equation,

$$
i \frac{\partial}{\partial t} \psi=\mathcal{H} \psi=\left[B(x, t)-\frac{J}{2} \frac{\partial^{2}}{\partial x^{2}}\right] \psi,
$$

for the evolution as a function of position, $x$. This is much less computationally expensive for many spins, yet still captures all of the essential features of our scheme. The continuum limit is important for practical cases, as the spins are typically separated by one, or a few, lattice sites, but the control fields are generated from surface gates and hence span many sites.

We first consider the case of a single spin guide. The form of the PT potential is $B(x, t)=-B_{0} \operatorname{sech}^{2}\left\{\left[x-x_{0}(t)\right] / w\right\}$, where the time dependence is determined by the moving center of the potential, $x_{0}(t)$. For simplicity in what follows, we set $J=1, B_{0}=1$, and $w=1$.

The magnon state is initialized as the lowest energy eigenstate of the moving PT potential, i.e.,

$$
\psi(x, t=0)=e^{i k x} \operatorname{sech}\left[x-x_{0}(0)\right] / \sqrt{2},
$$

where $x_{0}(0)$ is the center of the excitation (equivalent to the center of the spin guide), and the momentum $k$ is set to match the initial velocity of the spin guide. Throughout, we solve numerically for $\psi(x, t)$ and display $|\psi(x, t)|^{2}$.

To study the effectiveness of the channel, we examine the spin-guide fidelity $F$. The fidelity is given by the overlap between the initial wave function $\psi(x, t=0)$ and the excitation at the final time $t_{f}$ (shifted back to the original location, $\left.\psi^{*}\left[x+x_{0}\left(t_{f}\right), t=t_{f}\right]\right)$ :

$F=\left|\int_{-\infty}^{\infty} \psi(x, t=0) \psi^{*}\left[x+x_{0}\left(t_{f}\right), t=t_{f}\right] d x\right|^{2}$.

An important concept with optical fibers is bend loss, i.e., the extent to which an optical fiber can be bent before the mode ceases to be guided, and is therefore lost. The equivalent case is accelerating the magnon by investigating a single spin guide with a "corner." The center of the spin guide is given by

$$
x_{0}(t)=\sqrt{r+\tan ^{2} \theta\left(t-t_{f} / 2\right)^{2}}+\tan \theta\left(t-t_{f} / 2\right),
$$

where $r$ indicates the sharpness of the corner, $\theta$ is the angle through which the spin guide changes direction, and $t_{f}$ is the final time. The excitation is initially centered at position $x_{0}(0)$, with momentum $k=\partial x_{0}(t) /\left.\partial t\right|_{t=0}$.

Figure 2(a) utilizes Eq. (5) to show how the fidelity decreases with increasing angle through which the spin guide moves $\theta$. Two lines are shown: $r=0$ (discontinuous corner) always has lower fidelity than $r=2$ (smooth corner). As the corner is made more abrupt, the fidelity decreases, in accordance with our intuition from optical bend-loss results. The evolution with different examples of $r$ and $\theta$ is shown in Figs. 2(b)-2(d).

To complete the connection between spin guides and waveguides, we now consider two-port devices; i.e., we show how to create a "spin splitter" analogous to beam splitters. We first examine a spin splitter with a parallel component; see Fig. 3. The center of the potentials of the left and right spin guides are given by

$$
\begin{gathered}
x_{\text {right }}(t)=-x_{\text {left }}(t)= \begin{cases}f(x, t) & t<t_{f} / 2 \\
f\left(x, t_{f}-t\right) & t \geq t_{f} / 2,\end{cases} \\
f(x, t)=\sqrt{r+\left(4 m t+d-x_{l}\right)^{2} / 16}-m t+\left(d+x_{l}\right) / 4,
\end{gathered}
$$



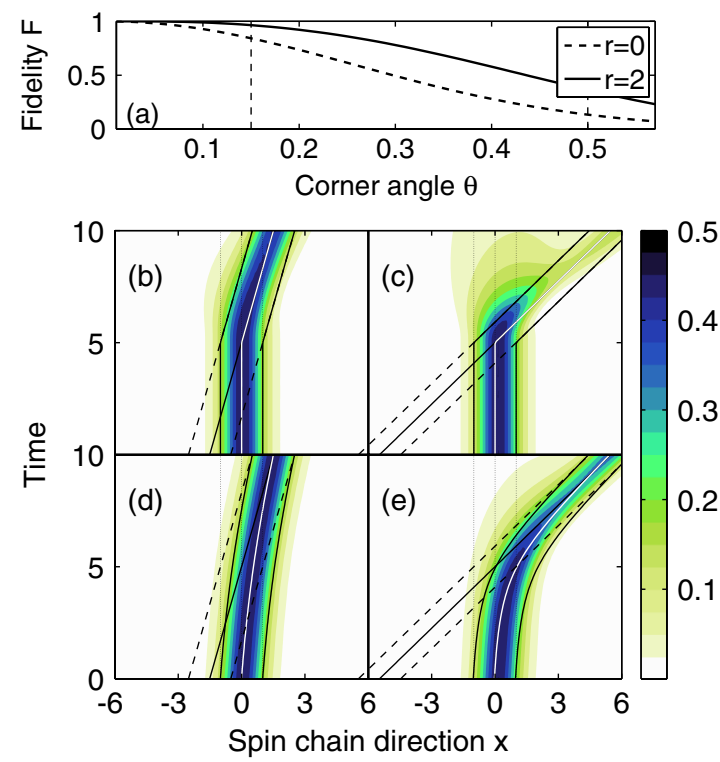

FIG. 2 (color online). (a) Fidelity as a function of corner angle $\theta$, for two values of $r: r=0$ (discontinuous corner, solid line) and $r=2$ (smooth corner, dashed line). Fidelity decreases with increasing angle $\theta$ in accordance with conventional optical bend loss. Four examples of magnon propagation are shown: (b) $r=0, \theta=0.15$ (fidelity 0.769), (c) $r=0, \theta=0.5$ (fidelity 0.106), (d) $r=2, \theta=0.15$ (fidelity 0.979), (e) $r=2, \theta=0.5$ (fidelity 0.401 ). In all cases, $t_{f}=10$.

where $m$ is the slope and $d$ is the separation between the parallel components of the spin guide. The magnon is initially in the left spin guide, i.e., $\psi\left(x=x_{\text {left }}(0), t=0\right)$, and the initial momentum is matched to the slope of the left spin guide $t=0$, that is, $k=\partial x_{\text {left }}(t) /\left.\partial t\right|_{t=0}$. In Fig. 3 the solid lines show $x_{\text {left }}$ and $x_{\text {right }}$, and the dashed lines show $x_{\text {left }} \pm 2$ and $x_{\text {right }} \pm 2$, which can be intuitively thought of as the "edge" of the spin guides. The excitation, after initially starting in the left spin guide, oscillates between spin guides, before leaving primarily through the right spin

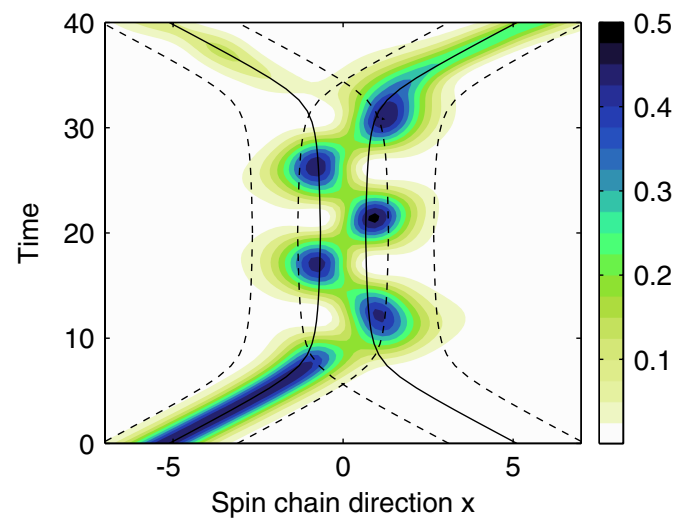

FIG. 3 (color online). Magnon evolution through a spin splitter with parallel component. Oscillatory behavior of the excitation between the spin guides is observed. Here, $r=0.5, d=1.2$, $m=0.3$. guide. This oscillation can be considered as the equivalent of evanescent tunneling as seen in coupled waveguides. As expected from coupled waveguides, the length of the parallel section compared to the oscillation frequency, set by the separation between spin guides, controls the final output distribution.

To quantify the spin-guide coupling, consider two parallel spin guides, separated by distance $d$, with an initial excitation which has zero momentum, $k=0$. The oscillation frequency into and out of the left waveguide is found by defining an effective Hamiltonian in the basis $\left\{\psi_{\text {left }}(x, t), \psi_{\text {right }}^{\prime}(x, t), \ldots\right\}$, where, $\psi_{\text {right }}^{\prime}(x, t)$ is determined by Gram-Schmidt orthonormalization relative to $\psi_{\text {left }}(x, t)$ and $\psi_{\text {right }}(x, t)$, which are given by Eq. (3). The resulting oscillation frequency is (given by the difference between the eigenvalues of the effective Hamiltonian),

$\Omega(d)=\frac{\operatorname{csch}^{2} d\left[\cosh 3 d+4 d \sinh d-\left(8 d^{2}+1\right) \cosh d\right]}{\cosh 2 d-2 d^{2}-1}$.

This function monotonically decreases from $\Omega(0)=$ $16 / 15$ to $\Omega(d) \approx 4 e^{-d}$ for large $d$.

A more practical form of spin splitter than that in Fig. 3 is an $X$ junction of spin guides. Two straight spin guides of length $2 x_{l}$ cross at an angle $\theta$, where $x_{R \rightarrow L}(t)=-x_{L \rightarrow R}(t)=x_{l} / 2-\tan (\theta / 2) t$. The initial excitation is placed in the left-to-right spin-guide, such that $x_{0}(0)=x_{L \rightarrow R}(0)$, with momentum $k_{L \rightarrow R}=\tan (\theta / 2)$.

The evolution for the $X$ junction is shown in Fig. 4, for time $t_{f}=x_{l} / \tan (\theta / 2)$. The relevant metrics for the spin splitter are shown in Fig. 4(a) as a function of spin-guide angle. The reflection and transmission coefficients $\mathrm{R}$ and $\mathrm{T}$, as defined by Eq. (4), and the total fidelity is $F_{\text {tot }}=\mathrm{R}+\mathrm{T}$. The behavior of $\mathrm{R}$ and $\mathrm{T}$ can be understood with regard to Landau-Zener theory $[24,25]$. When $\theta$ is large, the spin splitter shows a nonadiabatic crossing; therefore, the reflection (transmission) coefficient approaches zero (one) for large $\theta$, Fig. 4(c). Conversely, when $\theta$ is small, the spin guides approach an almost parallel state. As such, the excitation behaves similarly to Fig. 3, where the excitation oscillates between spin guides and therefore the fidelity depends strongly on $\theta$. In contrast to conventional LandauZener, this is not the adiabatic regime as the spin guides are forced to cross and the interaction time increases with decreasing angle so that oscillations are always observed.

An important spin-splitting ratio is 50/50 $(\mathrm{T}=\mathrm{R})$, and Fig. 4(a) shows many points where this ratio is approximately achieved, although with varying fidelity. An example, with $\theta=10^{-0.7976}$ is shown in Fig. 4(b), corresponding to the value of $\theta$ indicated by the circle intersecting the horizontal and vertical lines in Fig. 4(a), when $\mathrm{R} \approx \mathrm{T} \approx 0.491\left(F_{\text {tot }} \approx 0.982\right)$. These are slightly less than 0.5 due to scattering into nonbound modes. By choosing a sufficiently small $\theta$, one can generate a 50/50 spin splitter with a $\mathrm{T}$ and $\mathrm{R}$ arbitrarily close to 0.5 . 

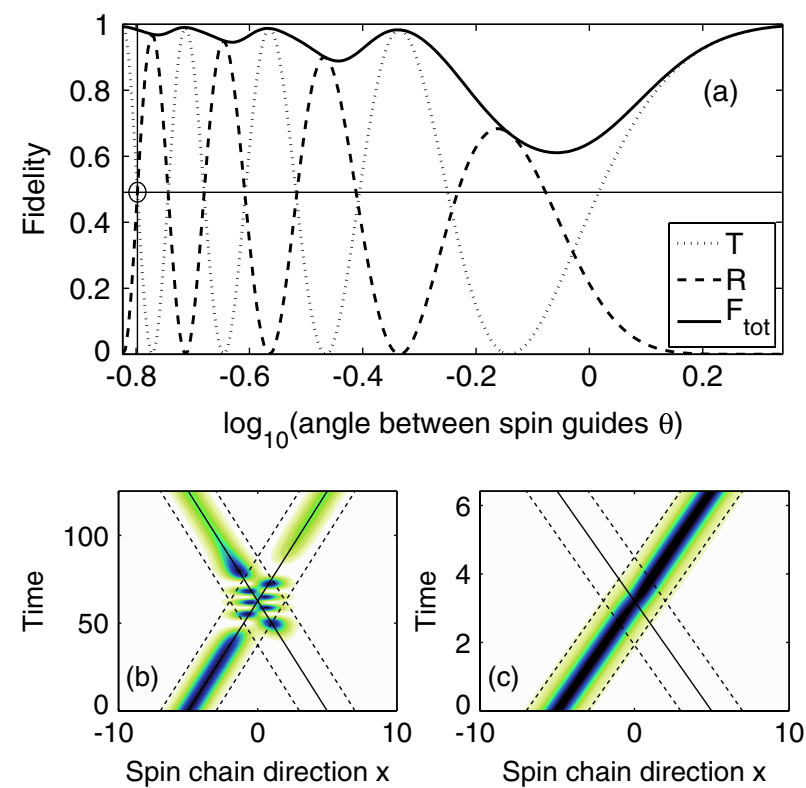

FIG. 4 (color online). (a) Fidelity and reflection and transmission coefficients for an $X$ junction spin splitter as a function of spin-guide angle. The excitation is initialized in the left-to-right spin guide and then evolved for time $t_{f}=x_{l} / \tan (\theta / 2)\left(x_{l}=10\right.$ fixed). The reflection and transmission coefficients show oscillations as expected from Landau-Zener theory. The spin-splitting fidelity is less than unity due to nonadiabaticity, although it asymptotes to one in both the small and large angle limits. (b) A $50 / 50$ spin splitter with $\theta=10^{-0.7976}$ (corresponding to $\mathrm{R} \approx \mathrm{T} \approx$ $0.491, F_{\text {tot }} \approx 0.982$, indicated by a circle at the intersection of a horizontal and a vertical line). (c) A nonadiabatic crossing with $\theta=10^{2.2}$. Note the different time scales in (b) and (c).

Finally, using our spin splitter we demonstrate the fundamental quantum mechanical characteristic of a beam splitter: the interference of paths due to their relative phase. Such a demonstration is necessary to prove that neither the spin guides nor the spin splitters degrade the quantum mechanical phase of the magnons. The phase is produced by introducing a Gaussian time-independent perturbation to the potential, which affects the right-to-left spin guide before the interaction takes place. This spatially localized potential is designed to mimic the effect of a phase shifter in a waveguide, and can equally be thought of as a prototype for the use of spin guides in interferometric sensing. The perturbation is a Gaussian of height $d$ and width $x_{l} / 10$, as indicated in Figs. 5(a)-5(e). When $d=0$, the depth of the two spin guides is identical, and no additional phase shift between the spin guides is incurred. A nonzero $d$ therefore imparts a phase shift. The wave function is initialized as $\psi(x, t=0)=\frac{1}{\sqrt{2}}\left[\psi_{L \rightarrow R}(x, 0)+\psi_{R \rightarrow L}(x, 0)\right]$. Figures 5(a)-5(e) show the evolution for specific values of $d$, illustrating the characteristic oscillations in the populations. These are indicative of conventional interferometric evolution. Note that Fig. 5(d), a phase shift of $\pi / 2$, shows magnon evolution that is essentially the time reversal of that presented in Fig. 4(b), as is required for unitary
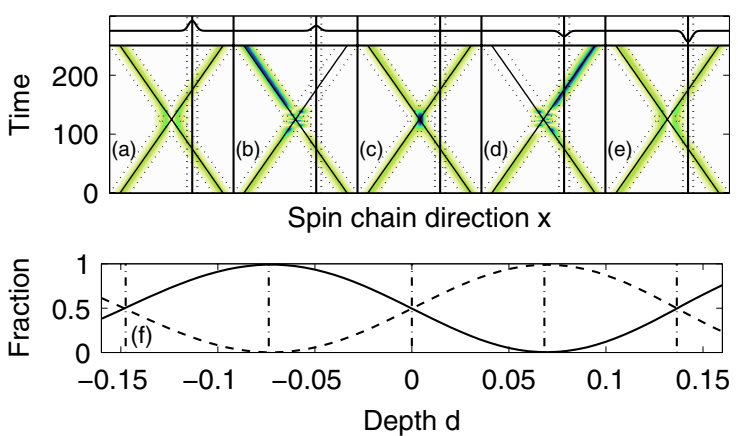

FIG. 5 (color online). Evolution of the spin splitter as a function of induced relative phase. (a)-(e) The magnon is initialized to an equally weighted superposition at time $t=0$. The relative phase between spin guides is varied by a localized, timeindependent Gaussian perturbation of depth $d$ in the potential in the right-to-left spin guide before the interaction takes place (shown as an inset along the top of the figure). The depths are (a) $d=-0.147$, (b) $d=-0.074$, (c) $d=0$, (d) $d=0.068$, (e) $d=0.136$. (f) The final spin-guide occupancies in the right-to-left (solid curve) and left-to-right (dashed curve) spin guides as a function of $d$. The vertical dot-dashed lines correspond to the simulations (a)-(e).

evolution in a beam-splitting device. The fraction in the left and right halves of the spin chain as a function of the depth $d$ is given in Fig. 5(f), again showing an obvious connection between the induced phase shift and the resultant output port of the spin splitter. This phase interference proves the quantum mechanical nature of the spin splitter, thereby completing the analogy between optical waveguides and the behavior of spin guides.

We have shown that a collective excitation within a linear spin chain can be confined and manipulated using localized field modulation. Specifically, we see that the space-time behavior of this one-dimensional excitation mimics effects traditionally observed with linear optics experiments (in two spatial dimensions). Using suitably chosen field modulations in space and time, we can replicate optical guiding modes, beam splitting, and even phase interference. This technique provides a new conceptual framework and method for controlling spin excitations using field modulation over distances much greater than the spin-spin separation.

M.I.M. acknowledges the support of the David Hay Foundation. C.D.H. acknowledges support from the Australian Research Council Centre of Excellence for Quantum Computation and Communication Technology (Project No. CE110001027). A. D. G. acknowledges the Australian Research Council for financial support (Project No. DP0880466).

[1] S. Bose, Contemp. Phys. 48, 13 (2007).

[2] D. Copsey, M. Oskin, F. Impens, T. Metodiev, A. Cross, F. T. Chong, I. L. Chuang, and J. Kubiatowicz, IEEE J. Sel. Top. Quantum Electron. 9, 1552 (2003). 
[3] R. Heule, C. Bruder, D. Burgarth, and V. M. Stojanovic, Phys. Rev. A 82, 052333 (2010).

[4] D. Burgarth, S. Bose, C. Bruder, and V. Giovannetti, Phys. Rev. A 79, 060305 (2009).

[5] A. Kay, Phys. Rev. A 84, 022337 (2011).

[6] A. S. Maloshtan and S. Ya. Kilin, Opt. Spektrosk. 108, 406 (2010) [Opt. Spectrosc. 108, 370 (2010)].

[7] D. Mogilevtsev, A. Maloshtan, S. Kilin, L. E. Oliveira, and S. B. Cavalcanti, J. Phys. B 43, 095506 (2010).

[8] D. Burgarth and S. Bose, Phys. Rev. A 71, 052315 (2005).

[9] G. M. Nikolopoulos, Phys. Rev. Lett. 101, 200502 (2008).

[10] M. Christandl, N. Datta, A. Ekert, and A. J. Landahl, Phys. Rev. Lett. 92, 187902 (2004).

[11] T. Ohshima, A. Ekert, D. K. L. Oi, D. Kaslizowski, and L. C. Kwek, arXiv:quant-ph/0702019.

[12] L. Zhou, Z. R. Gong, Y.X. Liu, C. P. Sun, and F. Nori, Phys. Rev. Lett. 101, 100501 (2008).

[13] J. Q. Liao, Z. R. Gong, L. Zhou, Y. X. Liu, C. P. Sun, and F. Nori, Phys. Rev. A 81, 042304 (2010).
[14] M. I. Makin, J. H. Cole, C. D. Hill, A. D. Greentree, and L. C. L. Hollenberg, Phys. Rev. A 80, 043842 (2009).

[15] F. Ladouceur and J. D. Love, Silica-Based Buried Channel Waveguides and Devices (Chapman and Hall, London, 1996).

[16] J. V. Kranendonk and J. H. V. Vleck, Rev. Mod. Phys. 30, 1 (1958).

[17] B. Culshaw, IEEE J. Sel. Top. Quantum Electron. 6, 1014 (2000).

[18] G. Pöschl and E. Teller, Z. Phys. 83, 143 (1933).

[19] N. Rosen and P. M. Morse, Phys. Rev. 42, 210 (1932).

[20] C. Eckart, Phys. Rev. 35, 1303 (1930).

[21] T. J. Osborne and N. Linden, Phys. Rev. A 69, 052315 (2004).

[22] A. J. Skinner, M. E. Davenport, and B. E. Kane, Phys. Rev. Lett. 90, 087901 (2003).

[23] J. M. Taylor, H. A. Engel, W. Dür, A. Yacoby, C. M. Marcus, P. Zoller, and M. D. Lukin, Nature Phys. 1, 177 (2005).

[24] L. D. Landau, Phys. Z. Sowjetunion 2, 46 (1932).

[25] C. Zener, Proc. R. Soc. A 137, 696 (1932). 